\title{
ATRIBUTOS EDÁFICOS DE CAVAS DE EXTRAÇÃO DE ARGILA APÓS CULTIVOS PUROS E CONSORCIADOS DE Eucalyptus spp. E Mimosa caesalpiniifolia BENTH (SABIÁ) E QUANTIFICAÇÃO DA PODA DE SABIÁ
}

\author{
Andrea Vita Reis Mendonça*, José Geraldo de Araújo Carneiro**, \\ Deborah Barroso Guerra***, Marcos P. Coutinho****, Josival Santos Souza***** \\ * Eng ${ }^{\text {a }}$. Florestal, Dr ${ }^{\mathrm{a}}$., Universidade Federal do Recôncavo da Bahia - avrmendonca@hotmail.com \\ ** Eng. Florestal, Dr, Universidade Estadual do Norte Fluminense - carneiro@uenf.br \\ ***Eng ${ }^{\mathrm{a}}$. Agrônoma, Dr ${ }^{\mathrm{a}}$. Universidade Estadual do Norte Fluminense - deborah@uenf.br \\ ****Eng. Florestal, M.Sc.; MMA - marcoscoutinho@mma.gov.br \\ *****Eng. Florestal, Dr., Bolsista DTI CNPq/Universidade Estadual de Feira de Santana - fragmentoflorestal@hotmail.com
}

Recebido para publicação: 23/02/2007 - Aceito para publicação: 03/03/2008

\begin{abstract}
Resumo
Este trabalho objetivou avaliar o efeito do plantio de eucalipto e de sabiá em sistemas puros e consorciados sobre características químicas do solo de cavas de extração de argila e quantificar biomassa e nutrientes fornecidos pelo material de plantas podadas de Mimosa caesalpiniifolia Benth (sabiá). Foram plantadas mudas de Eucalyptus camaldulensis, E. pellita, E. tereticornis, E. robusta e Mimosa caesalpiniifolia em plantios puros e consorciados. Realizaram-se análises do solo, por parcela, antes e 24 meses depois do plantio. As plantas de sabiá foram avaliadas quanto a sobrevivência, altura, diâmetro, área de copa e raízes finas. Quantificou-se a matéria seca do material podado aos 12 e 24 meses. Aos doze meses, determinou-se o teor de nutrientes, Na e C, nesse material. Entre os tratamentos testados, o mais promissor para recuperação do solo é o consórcio entre E. robusta + sabiá, por proporcionar aumento no teor de $\mathrm{C}$ no solo e contribuir para a redução da salinidade. Em todos os sistemas de plantio testados, houve redução de $\mathrm{K}$ no solo. Mimosa caesalpiniifolia apresenta menor crescimento quando consorciado com E. pellita. A biomassa seca total fornecida pela poda do sabiá em $\mathrm{t} \mathrm{ha}^{-1}$, aos 12 meses, no plantio puro de sabiá, foi de $3,6 \pm 0,5$, e no consorciado, de 1,3 $\pm 0,1$; aos 24 meses foi de $11,3 \pm 0,4$ no puro e de $4,6 \pm 0,1$ no consorciado.

Palavras-chave: Reabilitação de solo; Mimosa caesalpiniifolia; área degradada; raízes finas.
\end{abstract}

\begin{abstract}
Edaphic characteristics of a digging area for clay extraction after Eucalyptus spp. and Mimosa caesalpiniifolia Benth (sabia) in pure and mixed plantings and quantification of pruning of sabia. This work aimed to evaluate the effect of eucalipt and sabiá in pure and mixed plantings on chemical characteristics of the soil in a clay extraction area and quantify the biomass and nutrients from pruned material of Mimosa caesalpiniifolia Benth (sabiá) plants. Seedlings of Eucalyptus camaldulensis, E. pellita, E. robusta and Mimosa caesalpiniifolia were planted in both planting systems. Soil analysis were carried out in each plot before, and 24 months after the planting. The Mimosa caesalpiniifolia plants were evaluated regarding survival, height, diameter, crown area and fine roots. Dry biomass of the pruned material at the ages of 12 and 24 months was quantified. Nutrient contents of $\mathrm{Na}$ and $\mathrm{C}$ were determined. Among the treatments the most adequate for soil reabilitation is E. robusta + sabiá. This mixed planting stimulated increasing $\mathrm{C}$ content in the soil and reduction of the salinity. All plantings showed K reduction in the soil. Plants of Mimosa caesalpiniifolia showed lower growth when mixed with E. pellita. Total dry biomass from the pruning of the Mimosa caesalpiniifolia plants $\left(\mathrm{t} \mathrm{ha}^{-1}\right)$ at the age of 12 months of the pure planting was $3.6 \pm 0.5$ and in the mixed, $1.3 \pm 0.1$. At the age of 24 months, the values were $11.3 \pm 0.4$ in the pure planting and 4,6 \pm 0.1 in the mixed planting. Keywords: Soil rehabilitation; Mimosa caesalpiniifolia; degraded area; fine roots.
\end{abstract}

\section{INTRODUÇÃO}

O pólo ceramista de Campos do Goytacazes (RJ) é o segundo maior produtor de tijolos do Brasil, com produção mensal estimada de 90 milhões de peças (RAMOS et al., 2003). No processo de extração, são retiradas camadas do solo a profundidades variando de 1 a 4 m (VALICHESKI, 2004). 
Como resultado dessa exploração, pode ocorrer compactação do solo remanescente, afloramento de lençol freático e agravamento de problemas relativos à salinidade (RAMOS; MANZATTO, 2003; VALICHESKI, 2004), tornando comprometida a utilização da área para produção vegetal.

A seleção de espécies adaptadas às condições adversas e capazes de melhorar o solo é essencial para o sucesso do processo de mitigação das áreas resultantes do processo de extração de argila. Plantio de espécies perenes, principalmente espécies arbóreas, pode resultar em melhoria das características químicas, físicas e biológicas do solo.

A utilização de leguminosas fixadoras de $\mathrm{N}$ de rápido crescimento tem como finalidade aumentar a disponibilidade de $\mathrm{N}$ e acelerar a ciclagem de nutrientes (FORRESTER et al., 2005; FORRESTER et al., 2004), podendo também favorecer o aumento do estoque de C no solo, bem como melhorar suas características químicas (SCHIAVO, 2005; GARAY et al., 2003; VEZZANI et al., 2001; PARROTA, 1999; KUMAR et al., 1998; MAFRA et al., 1998; JAIYEOBA, 1996; TOKY; SINGH, 1995). Essas leguminosas podem ser plantadas em consórcio com espécies de maior interesse comercial, desempenhando a função de adubadeiras, principalmente quando podadas regularmente e o material proveniente da poda é espalhado na área (MAFRA et al., 1998). É desejável que a leguminosa também forneça produtos comercializáveis, a exemplo do sabiá, que tem potencial para ser utilizada como lenha (GONÇALVEZ et al., 1999) e moirão. A exploração dessa espécie para estacas, moirão, vara e lenha é uma importante atividade econômica no norte do Ceará (JÚNIOR LEAL; SILVA, 1999).

Os ceramistas e proprietários das cavas da região de Campos dos Goytacazes não se interessam por medidas puramente mitigadoras, buscando alternativas que resultem em retorno econômico. Dessa maneira, há a expectativa de que o consórcio de espécies arbóreas de interesse comercial com leguminosas fixadoras de $\mathrm{N}$, manejadas em sistema de podas, melhore as condições do solo e promova a utilização econômica e sustentável das áreas exploradas. Assim, o objetivo deste trabalho foi avaliar o efeito do plantio de Mimosa caesalpiniifolia Benth (sabiá), Eucalyptus camaldulensis Dehn., Eucalyptus pellita F. Muell., Eucalyptus tereticornis Smith e Eucalyptus robusta Smith, em sistemas puros e consorciados, sobre as características químicas do solo de cava de extração de argila e quantificar a biomassa e nutrientes fornecidos pelo material proveniente da poda das plantas de sabiá.

\section{MATERIAL E MÉTODOS}

Este estudo foi conduzido em uma cava de 1,5 ha, cuja profundidade média de exploração foi em torno de 1,5 m, no município de Campos dos Goytacazes (RJ) (2105' S, 41014' W, $11 \mathrm{~m}$ de altitude). Antes da exploração da argila, ocorrida entre janeiro de 1997 e agosto de 1998, a área estava sendo ocupada com plantio de cana-de-açúcar.

Segundo classificação de Köppen, o clima da região é tropical chuvoso (AW). Segundo dados fornecidos pela unidade meteorológica da Empresa de Pesquisa Agropecuária do Estado do Rio de Janeiro (PESAGRO-RIO), a temperatura média mensal do município variou nos últimos 10 anos entre $21,4{ }^{0} \mathrm{C}$ e $27,7{ }^{0} \mathrm{C}$, e a precipitação média anual foi de $852 \mathrm{~mm}$, com chuvas concentradas no período de outubro a dezembro. A precipitação apresentou valores de 90, 0, 40, 50, 20 e $0 \mathrm{~mm}$ nos seis primeiros meses após o plantio (maio a outubro), totalizando, aproximadamente, $200 \mathrm{~mm}$.

O solo original da área da cava em estudo é um Cambissolo Háplico Sódico Gleico Salino, com profundidade de lençol de 3,2 m (VALICHESKI, 2004). O solo remanescente da cava, segundo Valicheski (2004), é salino e sódico com saturação de sódio trocável superior a 15\%, com condutividade elétrica em pasta saturada superior a $4 \mathrm{dS} \mathrm{m} \mathrm{m}^{-1}$. Foi realizada, antes do plantio, a caracterização química, granulométrica e física do solo da cava. As análises químicas e granulométricas e a determinação da condutividade elétrica $(\mathrm{Ce})$ foram realizadas em amostras compostas de três sub-amostras, retiradas por parcela, nas profundidades de 0 a $20 \mathrm{~cm}$ e 20 a $40 \mathrm{~cm}$. Foram determinados: pH (em água), acidez potencial e C, segundo metodologia descrita pela EMBRAPA (1997); os teores de P, K e Na extraíveis pelo método de Mehlich ${ }^{-1}$; e $\mathrm{Ca}, \mathrm{Mg}$ e $\mathrm{Al}$ trocáveis por $\mathrm{KCl} 1 \mathrm{~mol} \mathrm{~L}^{-1}$. A composição granulométrica foi determinada pelo método da pipeta. Para a determinação da densidade, porosidade total, macro e microporosidade, foi coletada uma amostra indeformada por profundidade $(2,5-7,5 \mathrm{~cm}$ e 7,5-12,5 $\mathrm{cm})$ no centro de cada parcela, utilizando-se anéis volumétricos. As análises dessas características foram realizadas conforme procedimentos descritos por EMBRAPA (1997). A caracterização química está apresentada na tabela 1 . 
Tabela 1. Condutividade elétrica e caracterização química do solo da cava de extração de argila antes do plantio.

Table 1. Electric conductivity and chemical characterization of the ground of clay extraction before the plantation.

\begin{tabular}{|c|c|c|c|c|c|c|c|c|c|}
\hline \multirow{3}{*}{ Parâmetros } & \multicolumn{9}{|c|}{ Profundidade $0-20 \mathrm{~cm}$} \\
\hline & $\operatorname{Ce}(1: 1) *$ & $\mathbf{P}$ & pH & $\mathrm{C}$ & $\mathrm{Ca}$ & $\mathbf{M g}$ & $\mathbf{K}$ & $\mathbf{N a}$ & $\mathbf{H}+\mathbf{A l}$ \\
\hline & $\mathrm{dS} \mathrm{m}^{-1}$ & $\mathrm{mg} \mathrm{kg}^{-1}$ & $\mathrm{H}_{2} \mathrm{O}$ & $\%$ & \multicolumn{5}{|c|}{ cmolc dm dm $^{-3}$} \\
\hline EPM & 0,95 & 8,19 & 0,03 & 0,04 & 0,38 & 0,15 & 0,01 & 0,16 & 0,14 \\
\hline $\mathrm{CV} \%$ & 63,87 & 84,00 & 2,75 & 12,27 & 32,01 & 18,58 & 26,53 & 45,69 & 31,88 \\
\hline \multirow{3}{*}{ Parâmetros } & \multicolumn{9}{|c|}{ Profundidade $20-40 \mathrm{~cm}$} \\
\hline & Ce (1:1)* & $\mathbf{P}$ & pH & $\mathbf{C}$ & $\mathbf{C a}$ & Mg & $\mathbf{K}$ & $\mathbf{N a}$ & $\mathbf{H}+\mathbf{A l}$ \\
\hline & $\mathrm{dS} \mathrm{m}^{-1}$ & $\mathrm{mg} \mathrm{kg}^{-1}$ & $\mathrm{H}_{2} \mathrm{O}$ & $\%$ & \multicolumn{5}{|c|}{ cmolc dm-3 } \\
\hline Média & 6,99 & 34,77 & 6,07 & 1,41 & 4,32 & 3,30 & 0,09 & 1,72 & 1,35 \\
\hline
\end{tabular}

A composição granulométrica do solo da cava de extração de argila, antes do plantio na camada de 0 a $20 \mathrm{~cm}$, foi $40,55 \% \pm 1,54 \%$ de argila, $27,39 \% \pm 2,54 \%$ de areia e $32,06 \% \pm 1,43 \%$ de silte, e na camada de 20 a 40 foi $27,43 \% \pm 2,21 \%$ de argila, $50,1 \% \pm 4,03 \%$ de areia e $22,47 \% \pm 2,20 \%$ de silte. A densidade da cava de extração de argila, antes do plantio, nas profundidades de 2,5 a $7,5 \mathrm{~cm}$ e de 7,5 a $12,5 \mathrm{~cm}$, foram de $1,24 \mathrm{Mg} \mathrm{m}^{-3} \pm 0,02 \mathrm{Mg} \mathrm{m}^{-3}$ e $1,31 \mathrm{Mg} \mathrm{m}^{-3} \pm 0,02 \mathrm{Mg} \mathrm{m}^{-3}$, respectivamente.

$\mathrm{Na}$ ocasião da exploração da argila, a camada superficial $(20 \mathrm{~cm})$ foi retirada e separada. Posteriormente, esse solo separado foi distribuído na cava com trator de esteira. Após esse procedimento, a cava foi abandonada por dois anos e manteve-se totalmente sem vegetação até a ocasião do plantio, ou seja, não havia indícios de regeneração natural.

$\mathrm{Na}$ época do plantio, o solo foi gradeado e as parcelas foram delimitadas, sendo em seguida coletadas amostras de solo para análise, conforme descrito anteriormente. O plantio foi realizado em covas de $20 \mathrm{~cm}$ de profundidade, no espaçamento $3 \times 2 \mathrm{~m}$, não sendo realizadas adubações de plantio e de cobertura.

As mudas utilizadas no plantio foram produzidas a partir de sementes, sendo as de sabiá obtidas na CNPAB-EMBRAPA (Seropédica, RJ) e as de eucalipto no Instituto de Pesquisas e Estudos Florestais (IPEF). As mudas de eucalipto foram produzidas em tubetes de $50 \mathrm{~cm}^{3}$, e as de sabiá em tubetes de 288 $\mathrm{cm}^{3}$, em casa de vegetação da Universidade Estadual do Norte Fluminense Darcy Ribeiro. O período de produção das mudas foi de três meses. As sementes de sabiá foram inoculadas com rizóbio específico à espécie leguminosa (BR3405/BR3451), obtido no CNPAB-EMBRAPA (Seropédica, RJ).

$\mathrm{O}$ experimento foi composto por nove tratamentos, dispostos em blocos casualizados, com quatro repetições. Cada parcela foi constituída de 36 plantas, dispostas em seis linhas. Os tratamentos foram: E. camaldulensis, E. pellita, E. tereticornis, E. robusta, sabiá, E. camaldulensis + sabiá, E. pellita + sabiá, E. tereticornis + sabiá, E. robusta + sabiá. Nas parcelas consorciadas, as mudas foram plantadas em linhas alternadas, sendo as linhas pares compostas por eucalipto e as ímpares por sabiá.

Durante o primeiro mês de plantio no campo, as mudas foram irrigadas duas vezes por semana, recebendo, em média, 2 litros de água por muda. Durante o segundo mês, foram irrigadas uma vez por semana, recebendo a mesma quantidade de água. Para controlar a competição com a vegetação espontânea, foi realizado coroamento de todas as mudas aos sete e dez meses após o plantio.

Aos 24 meses após o plantio, foram determinados os teores de nutrientes e de $\mathrm{C}$ e a condutividade elétrica do solo, conforme amostragem e metodologias de análise adotadas antes do plantio. 
As plantas de sabiá foram avaliadas quanto à sobrevivência, à altura e ao diâmetro ao nível do solo (DAS) aos 12 e 24 meses, sendo a área de copa medida aos 12 meses. A mensuração das características biométricas foi realizada nas linhas ímpares, totalizando 18 plantas por parcela.

Aos 12 e 24 meses, foram realizadas podas das plantas de sabiá, e o material proveniente dessa poda foi espalhado entre as linhas de plantio no interior da parcela. A poda foi conduzida no sentido de preservar todas as hastes retilíneas, permanecendo de 1 a 3 hastes por planta, das quais foram retirados todos os galhos. Para quantificação da biomassa seca do material podado, foram utilizadas duas plantas por parcela, selecionadas aleatoriamente. $\mathrm{O}$ material foi dividido em folhas e galhos e, posteriormente, foi determinada a biomassa fresca. Uma amostra de peso conhecido desse material foi colocada em estufa de circulação forçada de ar, à temperatura constante de $65{ }^{\circ} \mathrm{C}$, por 72 horas, e pesada para determinação da biomassa seca incorporada ao sistema. Posteriormente, esse material foi analisado para a determinação dos teores de N (Nessler), P (colorimetria), K (fotometria de chama), S (turbidimetria), C (digestão com dicromato de potássio), $\mathrm{Ca}, \mathrm{Mg}, \mathrm{Fe}, \mathrm{Cu}, \mathrm{Zn}$ e $\mathrm{Mn}$ (espectrofotometria de absorção atômica), segundo Jones Júnior et al. (1991) e Malavolta et al. (1997). É importante ressaltar que a análise nutricional nos tecidos provenientes da poda foi realizada apenas para o material coletado aos 12 meses.

Aos 24 meses de idade, foram avaliados a área superficial e o comprimento de raízes finas $(\leq 2$ $\mathrm{mm})$ das plantas de sabiá. As coletas foram realizadas com o auxílio de um trado $(\phi=2,5 \mathrm{~cm})$, na profundidade de 0 a $10 \mathrm{~cm}$. Em cada repetição foram tomadas duas amostras, retiradas a $20 \mathrm{~cm}$ de distância do tronco das plantas de altura média, na linha de plantio do eucalipto. As amostras foram acondicionadas em sacolas plásticas e armazenadas em "freezer". As raízes foram separadas do solo por coleta manual a seco e colocadas, aleatoriamente, entre duas placas de vidro de $2 \mathrm{~mm}$ de espessura, $21 \mathrm{~cm}$ de largura e $30 \mathrm{~cm}$ de comprimento. Em seguida, obteve-se a imagem por meio de scanner, que foi processada utilizando-se o programa QuantRoot, desenvolvido pelo Departamento de Solos da Universidade Federal de Viçosa, para a determinação de comprimento, área superficial e diâmetro de raízes.

Para analisar o efeito dos tratamentos sobre os teores de $\mathrm{C}, \mathrm{pH}, \mathrm{Al}, \mathrm{H}+\mathrm{Al}, \mathrm{Na}$, condutividade elétrica no extrato de saturação 1:1, Na, P, K, Ca e Mg no solo da cava, foi utilizado o teste t para dados emparelhados (SNEDECOR; COCHRAN, 1989), considerando-se os resultados da análise do solo antes e depois do plantio.

Foram ajustados modelos para estimar a massa seca de folhas, galhos e a massa seca total, proveniente da poda, em função do diâmetro à altura do solo (DAS), da altura (H) e da área de copa (AC). Os modelos testados foram: MS $=\beta_{0}+\beta_{1} \mathrm{DAS}+\mathrm{e}_{\mathrm{i}} ; \mathrm{MS}=\beta_{0}+\beta_{1} \mathrm{AC}+\mathrm{e}_{\mathrm{i}} ; \mathrm{MS}=\beta_{0}+\beta_{1} \mathrm{DAS}+\beta_{2} \mathrm{H}+\beta_{3} \mathrm{AC}$ $+\mathrm{e}_{\mathrm{i}} ; \mathrm{MS}=\beta_{0}+\beta_{1} \mathrm{DAS}+\beta_{2} \mathrm{H}+\mathrm{e}_{\mathrm{i}} ; \mathrm{MS}=\beta_{0}+\beta_{1} \mathrm{DAS}+\beta_{2} \mathrm{AC}+\mathrm{e}_{\mathrm{i}} ; \mathrm{MS}=\beta_{0}+\beta_{1} \mathrm{DAS} * \mathrm{H}+\mathrm{e}_{\mathrm{i}} ; \mathrm{MS}=\beta_{0}$ $+\beta_{1} \mathrm{AC}+\beta_{2} \mathrm{DAS}^{*} \mathrm{H}+\mathrm{e}_{\mathrm{i}} ; \mathrm{MS}=\beta_{0} * \mathrm{DAS}^{\beta 1}+\mathrm{e}_{\mathrm{i}} ; \mathrm{MS}=\beta_{0} * \mathrm{AC}^{\beta 1}+\mathrm{e}_{\mathrm{i}}$ (em que: $\mathrm{MS}=$ massa seca - folhas, galhos ou total; $\beta_{\mathrm{i}}=$ parâmetros estimados; $\mathrm{DAS}=$ diâmetro ao nível do solo $-\mathrm{mm} ; \mathrm{H}=$ altura $-\mathrm{cm} ; \mathrm{AC}=$ área de copa $-\mathrm{m}^{2}$; e $\mathrm{e}_{\mathrm{i}}=$ resíduo). Para a seleção das equações obtidas, utilizou-se a distribuição gráfica dos resíduos padronizados, o teste t para os parâmetros estimados (modelos com duas ou mais variáveis), o desvio padrão e o coeficiente de determinação $\left(\mathrm{R}^{2}\right)$.

Selecionado o melhor modelo, estimou-se a biomassa seca por área (folhas, galhos e total) dos tratamentos com a presença de sabiá, obtendo-se a biomassa seca por planta e, posteriormente, a biomassa seca por parcela (= $\left.\sum \mathrm{MS}\right)$, extrapolando para hectare. Com os dados de biomassa seca de folhas e galhos, estimou-se o aporte de C, Na e nutrientes por unidade de área (hectare).

Para analisar a sobrevivência, o DAS, a altura, a área de copa, o comprimento e a área de raízes finas, a biomassa seca de folha $\left(\mathrm{t} \mathrm{ha}^{-1}\right)$, a biomassa seca de galho $\left(\mathrm{t} \mathrm{ha}^{-1}\right)$, a biomassa seca total $\left(\mathrm{t} \mathrm{ha}^{-1}\right)$ e a quantidade de $\mathrm{C}, \mathrm{Na}$ e nutrientes fornecidos pela poda do sabiá, realizou-se análise de variância para delineamento em blocos casualizados. Considerou-se, para esse fim, os cinco tratamentos com a presença de sabiá (sabiá puro, E. camaldulensis + sabiá, E. pellita + sabiá, E. tereticornis + sabiá e E. robusta + sabiá) e, posteriormente, aplicou-se o teste Tukey $(\alpha=0,05)$. Antes da análise de variância, os dados foram analisados quanto à homocedasticidade, pelo teste de Cochran (SNEDECOR; COCHRAN, 1989), e quanto à distribuição normal dos resíduos, pelo teste de Lilliefors (CAMPOS, 1979).

$\mathrm{O}$ intervalo de confiança foi calculado para a biomassa seca e para a quantidade de $\mathrm{C}$, de nutrientes e de $\mathrm{Na}$ fornecida pelo material proveniente da poda. 


\section{RESULTADOS E DISCUSSÃO}

\section{Alterações no solo da cava de extração de argila}

As espécies testadas proporcionaram alterações em algumas das características edáficas avaliadas, conforme os resultados do teste t para dados emparelhados, comparando o solo antes e depois do plantio. Estão apresentados, na tabela 2, apenas os resultados das alterações significativas. Os tratamentos em que não se constataram diferenças significativas estão sinalizando que as características avaliadas não sofreram alterações, após 24 meses de plantio, estando com valores equivalentes aos encontrados antes da implantação do experimento (Tabela 1).

Constatou-se, neste estudo, que o $\mathrm{C}$ do solo aumentou no plantio consorciado do $E$. camaldulensis + sabiá (profundidade 0-20), no de E. robusta + sabiá (profundidade 20-40) e no plantio puro de sabiá (profundidade 0-20), conforme pode-se observar na tabela 2. Esses resultados evidenciam que o plantio de sabiá sob podas freqüentes contribui para aumentar o estoque de matéria orgânica do solo, favorecendo a sua reabilitação.

É importante ressaltar que, quando o sabiá foi consorciado com E. pellita ou com E. tereticornis, não foram verificadas alterações significativas na quantidade de $\mathrm{C}$ do solo. Embora não tenha sido avaliada neste trabalho, a taxa de decomposição de serapilheira, os resultados encontrados levam a supor que a serapilheira formada por essas combinações de espécies apresentou maior taxa de decomposição em relação às combinações de E. camaldulensis e E. robusta com o sabiá. Uma serapilheira de mais fácil decomposição resulta em menor acúmulo de C no solo, considerando-se uma mesma granulometria, quando comparada a outra mais resistente a agentes decompositores (GAMA RODRIGUES et al., 1999). Entretanto, destaca-se, neste estudo, que tanto nos plantios puros como nos consorciados não houve redução do teor de $\mathrm{C}$ inicial após 24 meses, sendo garantida a manutenção da reserva de carbono do solo.

$\mathrm{O}$ aumento do $\mathrm{C}$ no solo na camada de 20 a $40 \mathrm{~cm}$ de profundidade, proporcionado pelo consórcio entre E. robusta e sabiá, pode, também, ter sido favorecido pela dinâmica do sistema radicular do E. robusta. Os resultados apresentados por Mendonça (2006), no sistema consorciado, mostraram que E. robusta apresentou maior área e comprimento de raízes finas. Mafra et al. (1998) também observaram aumento da reserva de $\mathrm{C}$ do solo em resposta ao cultivo em aléias, após nove anos de implantação, tendo como espécie adubadeira a Leucaena leucocephala, sendo esses resultados compatíveis com os encontrados no presente estudo.

Diferentemente do procedimento adotado neste estudo, no qual se comparou os resultados de amostras de solo, retiradas de uma mesma área, só que em tempos diferentes, antes e depois do plantio, nos trabalhos sobre alterações das características do solo em função de cultivos florestais, normalmente, são tomadas como referências amostras de solo de áreas com vegetação nativa preservada ou de locais próximos à área em estudo.

Contrariando os resultados encontrados neste estudo, Mishra et al. (2003) constataram que o plantio puro de E. tereticornis proporcionou aumento no teor de $\mathrm{C}$ na profundidade de $10 \mathrm{~cm}$, aos três anos de idade, em um solo sódico na Índia, tendo como referência o solo de uma área improdutiva. Zinn et al. (2002) observaram em um Latossolo Vermelho Argiloso com um povoamento de E. camaldulensis, localizado no norte de Minas Gerais, redução no teor de $\mathrm{C}$ na profundidade de $5 \mathrm{~cm}$, quando comparado a uma área de cerrado nativo.

Diferentemente do esperado, na profundidade de 0-20 cm, constatou-se aumento do $\mathrm{pH}$ nos plantios de E. camaldulensis, E. tereticornis, E. tereticornis + sabiá e E. robusta + sabiá e de $20-40 \mathrm{~cm}$ para E. tereticornis, E. tereticornis + sabiá e sabiá puro (Tabela 2). Santiago (2005) também observou aumento de $\mathrm{pH}$, após um ano de plantio, em resposta ao plantio de sesbânia + E. robusta e sesbânia $+E$. tereticornis em solo de cava de extração de argila na região de Campos dos Goytacazes (RJ). O aumento de $\mathrm{pH}$ pode ter sido favorecido por ânions orgânicos presentes na serrapilheira dos plantios. Noble et al. (1996) incubaram um solo por oito semanas utilizando serrapilheira de 16 espécies florestais e observaram aumento de $\mathrm{pH}$. Contudo, Mishra et al. (2003) observaram decréscimo de $\mathrm{pH}$ em plantio puro de E. tereticornis, aos três, seis e nove anos, em solo sódico.

A acidez potencial $(\mathrm{H}+\mathrm{Al})$ aumentou nos plantios puros de E. camaldulensis $(0-20 \mathrm{~cm})$, sabiá (0-20) e E. robusta (20-40) e nos plantios consorciados E. tereticornis + sabiá $(20-40 \mathrm{~cm})$ e E. robusta + sabiá $(20-40 \mathrm{~cm})$, conforme apresentado na tabela 2. Em pH acima de 6, condição observada na maioria das parcelas deste experimento, existe acidez ativa e acidez não trocável, mas não ocorre acidez trocável, 
uma vez que todo o alumínio já foi precipitado na forma de $\mathrm{Al}(\mathrm{OH})_{3}$, não havendo efeito negativo sobre as plantas. Alvarenga; Davide (1999) constataram aumento da acidez potencial numa área de reforma de E. camaldulensis, atribuindo esse efeito ao processo de decomposição dos resíduos vegetais resultante do plantio anterior.

Tabela 2. Resultados do teste $t$ (dados emparelhados) para os tratamentos que apresentaram diferenças significativas, comparando características químicas do solo da cava de extração de argila antes e 24 meses após o plantio.

Table 2. Results of $t$ test (paired data) for the treatments with significant difference regarding chemical characteristics of the soil of a digging area for clay extraction before and 24 months after the planting.

\begin{tabular}{|c|c|c|c|c|c|}
\hline Tratamento & Profundidade & Característica & Antes & Depois & t cal \\
\hline Sabiá & $0-20 \mathrm{~cm}$ & $\mathrm{C}$ & 1,6 & 2,0 & 3,9 \\
\hline E. camaldulensis + sabiá & $0-20 \mathrm{~cm}$ & $\mathrm{C}$ & 1,8 & 2,1 & 3,5 \\
\hline E. robusta + sabiá & $20-40 \mathrm{~cm}$ & $\mathrm{C}$ & 1,0 & 1,4 & 3,2 \\
\hline Sabiá & $0-20 \mathrm{~cm}$ & $\mathrm{H}+\mathrm{Al}$ & 2,7 & 3,6 & 4,1 \\
\hline E. camaldulensis & $0-20 \mathrm{~cm}$ & $\mathrm{H}+\mathrm{Al}$ & 2,0 & 3,1 & 3,3 \\
\hline Sabiá & $20-40 \mathrm{~cm}$ & $\mathrm{H}+\mathrm{Al}$ & 1,6 & 3,2 & 7,5 \\
\hline E. pellita & $20-40 \mathrm{~cm}$ & $\mathrm{H}+\mathrm{Al}$ & 1,9 & 3,3 & 3,7 \\
\hline E. tereticornis + sabiá & $20-40 \mathrm{~cm}$ & $\mathrm{H}+\mathrm{Al}$ & 0,6 & 2,4 & 5,0 \\
\hline E. robusta + sabiá & $20-40 \mathrm{~cm}$ & $\mathrm{H}+\mathrm{Al}$ & 1,0 & 3,1 & 3,4 \\
\hline E. robusta & $20-40 \mathrm{~cm}$ & $\mathrm{H}+\mathrm{Al}$ & 1,7 & 2,8 & 6,0 \\
\hline E. tereticornis & $0-20 \mathrm{~cm}$ & $\mathrm{Ce}$ & 7,3 & 3,6 & 3,6 \\
\hline E. robusta + sabiá & $0-20 \mathrm{~cm}$ & $\mathrm{Ce}$ & 6,6 & 3,1 & 3,8 \\
\hline Sabiá & $0-20 \mathrm{~cm}$ & $\mathrm{pH}$ & 6,0 & 6,5 & 5,1 \\
\hline E. camaldulensis & $0-20 \mathrm{~cm}$ & $\mathrm{pH}$ & 5,9 & 6,4 & 3,7 \\
\hline E. tereticornis & $0-20 \mathrm{~cm}$ & $\mathrm{pH}$ & 6,0 & 6,5 & 7,0 \\
\hline E. tereticornis + sabiá & $0-20 \mathrm{~cm}$ & $\mathrm{pH}$ & 6,1 & 6,7 & 3,5 \\
\hline E. robusta + sabiá & $0-20 \mathrm{~cm}$ & $\mathrm{pH}$ & 6,1 & 6,8 & 6,3 \\
\hline E. tereticornis & $20-40 \mathrm{~cm}$ & $\mathrm{pH}$ & 6,0 & 6,6 & 3,8 \\
\hline E. tereticornis + sabiá & $20-40 \mathrm{~cm}$ & $\mathrm{pH}$ & 6,2 & 7,0 & 3,8 \\
\hline E. tereticornis & $0-20 \mathrm{~cm}$ & $\mathrm{Na}$ & 1,8 & 1,2 & 3,8 \\
\hline Sabiá & $0-20 \mathrm{~cm}$ & $\mathrm{Na}$ & 2,3 & 1,2 & 3,3 \\
\hline Sabiá & $0-20 \mathrm{~cm}$ & $\mathrm{~K}$ & 0,13 & 0,06 & 4,7 \\
\hline E. camaldulensis & $0-20 \mathrm{~cm}$ & $\mathrm{~K}$ & 0,13 & 0,05 & 5,3 \\
\hline E. pellita & $0-20 \mathrm{~cm}$ & $\mathrm{~K}$ & 0,16 & 0,06 & 5,6 \\
\hline E. tereticornis & $0-20 \mathrm{~cm}$ & $\mathrm{~K}$ & 0,13 & 0,06 & 10,6 \\
\hline Tratamento & Profundidade & Característica & Antes & Depois & t cal \\
\hline E. robusta & $0-20 \mathrm{~cm}$ & $\mathrm{~K}$ & 0,16 & 0,05 & 6,8 \\
\hline E. camaldulensis + sabiá & $0-20 \mathrm{~cm}$ & $\mathrm{~K}$ & 0,11 & 0,05 & 3,6 \\
\hline E. pellita + sabiá & $0-20 \mathrm{~cm}$ & $\mathrm{~K}$ & 0,15 & 0,08 & 3,7 \\
\hline E. tereticornis + sabiá & $0-20 \mathrm{~cm}$ & $\mathrm{~K}$ & 0,13 & 0,05 & 3,3 \\
\hline E. robusta + sabiá & $0-20 \mathrm{~cm}$ & $\mathrm{~K}$ & 0,13 & 0,04 & 6,5 \\
\hline Sabiá & $20-40 \mathrm{~cm}$ & $\mathrm{~K}$ & 0,08 & 0,04 & 6,4 \\
\hline E. camaldulensis & $20-40 \mathrm{~cm}$ & $\mathrm{~K}$ & 0,10 & 0,04 & 4,0 \\
\hline E. pellita & $20-40 \mathrm{~cm}$ & $\mathrm{~K}$ & 0,11 & 0,04 & 3,3 \\
\hline E. tereticornis & $20-40 \mathrm{~cm}$ & $\mathrm{~K}$ & 0,08 & 0,04 & 3,2 \\
\hline E. robusta & $20-40 \mathrm{~cm}$ & $\mathrm{~K}$ & 0,08 & 0,03 & 8,6 \\
\hline E. camaldulensis + sabiá & $20-40 \mathrm{~cm}$ & $\mathrm{~K}$ & 0,09 & 0,04 & 3,7 \\
\hline E. tereticornis + sabiá & $20-40 \mathrm{~cm}$ & $\mathrm{~K}$ & 0,08 & 0,03 & 5,3 \\
\hline E. robusta + sabiá & $20-40 \mathrm{~cm}$ & $\mathrm{~K}$ & 0,09 & 0,04 & 4,1 \\
\hline E. pellita + sabiá & $20-40 \mathrm{~cm}$ & $\mathrm{Mg}$ & 4,60 & 4,20 & 3,2 \\
\hline E. pellita + sabiá & $0-20 \mathrm{~cm}$ & CTC efetiva & 15,29 & 13,98 & 3,7 \\
\hline
\end{tabular}

Valor de $\mathrm{t}$ tabelado a $95 \%$ de confiança igual a 3,2. 
A condutividade elétrica do solo foi reduzida na profundidade de $0-20 \mathrm{~cm}$, no plantio de $E$. tereticornis puro e de E. robusta + sabiá, assim como o teor de Na nos plantios puros de E. tereticornis e de sabiá (Tabela 2). Resultado semelhante foi observado por Mishra et al. (2003) para o E. tereticornis. Segundo esses autores, a redução da condutividade elétrica e do Na no solo deve-se à maior lixiviação de sais devido à melhoria da estrutura do solo proporcionada pelas raízes. Outra provável explicação é o acúmulo de $\mathrm{Na}$ pelas plantas, principalmente em plantios de E. tereticornis, considerando que essa espécie apresenta indícios de utilizar estratégias de inclusão para tolerar salinidade (MENDONÇA, 2006). Para áreas com problemas relativos à salinidade, plantios que promovam a redução da condutividade elétrica e do teor de $\mathrm{Na}$ no solo são desejável.

No solo, o $\mathrm{K}$ teve seu valor reduzido com o plantio de todas as espécies em estudo, nos dois sistemas de plantio e nas duas profundidades, com exceção do E. pellita + sabiá na profundidade de 20-40 $\mathrm{cm}$ (Tabela 2). Essa redução do $\mathrm{K}$ pode estar relacionada à retenção desse nutriente na biomassa da planta e/ou à lixiviação, considerando-se que o K é facilmente lixiviado (TOKY; RAMAKRISHNAN, 1982), bem como à adsorção em complexos orgânico-metálicos. Toky e Singh (1995), avaliando a dinâmica de nutrientes em plantios de E. tereticornis, Leucaena leucocephala e Acacia nilótica, observaram que a retenção de $\mathrm{K}$ na biomassa das plantas foi alta e que o retorno desse nutriente pela serapilheira foi baixo. Mello et al. (1995) demonstraram a existência de alta correlação entre os teores de K extraídos do solo pelos ácidos orgânicos de baixo peso molecular e pelo ácido nítrico, com a absorção desse nutriente pelas plantas de eucalipto. Os ácidos orgânicos são comumente exsudados pelas raízes das plantas, permanecendo em alta concentração na rizosfera (SONG; HUANG, 1988). Esses ácidos apresentam alta capacidade de interagir com metais, formando complexos orgânico-metálicos na solução do solo, reduzindo a concentração dos nutrientes e induzindo suas liberações pelos minerais do solo (MELO et al., 2005).

Santiago (2005), trabalhando com plantios puros de E. camaldulensis, E. pellita, E. robusta e $E$. tereticornis e consórcios de cada uma dessas espécies com sesbânia, encontrou, aos 12 meses de idade, resultados diferentes dos resultados deste trabalho, sendo observado que o teor de $\mathrm{K}$ do solo não foi significativamente alterado. Mishra et al. (2003) observaram aumento no teor de K em solo sob plantio de E. tereticornis, entretanto esse aumento foi observado apenas nas camadas mais profundas, 60 e $150 \mathrm{~cm}$ de profundidade, indicando a tendência de lixiviação desse nutriente.

$\mathrm{O}$ teor de $\mathrm{Mg}$ no solo foi reduzido apenas no plantio misto de E. pellita + sabiá, na profundidade de 20-40 cm (Tabela 2). Resultado semelhante foi encontrado por Santiago (2005), que constatou redução desse elemento no plantio consorciado de E. pellita + sesbânia.

A capacidade de trocas de cátions efetiva (CTC efetiva) foi reduzida apenas no plantio consorciado de E. pellita + sabiá, na profundidade de 0-20 cm (Tabela 2), enquanto que nos demais tratamentos não foram verificadas alterações significativas para essa característica. Diferentemente dos resultados encontrados neste estudo, Santiago (2005), em plantios puros de eucalipto e consorciados com sesbânia, e Parrota (1999), em experimento com plantios puros e consorciados de E. robusta, Casuarina equisetifolia e Leucena leucocephala, observaram, na maioria dos tratamentos testados, redução da CTC efetiva.

\section{Avaliação da sobrevivência, características dendrométricas e raízes finas de sabiá}

A sobrevivência das plantas de sabiá, aos 12 e 24 meses, foi equivalente, não sendo afetada pelos tratamentos testados. Aos 12 meses, o sistema de plantio não influenciou o diâmetro à altura do solo (DAS) e a altura do sabiá, enquanto que a área de copa (AC) média por planta foi maior no plantio puro, diferindo apenas no plantio consorciado com E. pellita. Aos 24 meses, o DAS e a altura das plantas de sabiá foi superior no plantio puro, mas diferiu apenas do plantio consorciado com E. pellita (Tabela 3). Este resultado pode ser um indicativo de que o sabiá tem seu crescimento prejudicado quando consorciado com E. pellita, ocorrendo competição interespecífica.

Segundo Paulino et al. (2002), o plantio de sabiá apresentou alta sobrevivência e crescimento em pastagem degradada, apresentando, aos 24 meses, $87 \%$ de sobrevivência e 3,26 m de altura, resultados inferiores aos constatados neste estudo.

O sistema de plantio não afetou a produção de raízes finas do sabiá aos 24 meses de idade (Tabela 4). Considerando os resultados das características dendrométricas, era de se esperar que, quando consorciado com E. pellita, o sabiá apresentasse maior área e comprimento de raízes finas, como reflexo da competição interespecífica, uma vez que a menor disponibilidade de recursos, normalmente, estimula a produção dessas raízes (KEITH et al., 1997; GIARDINA; RYAN, 2002; MISRA et al., 1998; SCHMID; 
KAZDA, 2002; GONÇALVEZ, 1995), fato observado para E. robusta consorciado com Mimosa caesalpiniifolia (MENDONÇA, 2006). Entretanto, embora o sabiá tenha apresentado maior área e comprimento de raízes finas quando consorciado com E. pellita, a análise estatística comprovou que essa diferença não é significativa.

Tabela 3. Valores médios de sobrevivência e características dendrométricas de plantas de sabiá, aos 12 e 24 meses após o plantio.

Table 3. Average values of survival and biometric characteristics of plants of sabia, at 12 and 24 months after the plantation.

\begin{tabular}{|c|c|c|c|c|c|c|}
\hline \multirow[b]{2}{*}{ Tratamentos } & \multicolumn{4}{|c|}{12 meses } & \multicolumn{2}{|c|}{24 meses } \\
\hline & \% Sobrevivência & $\begin{array}{c}\text { DAS } \\
(\mathrm{mm})\end{array}$ & $\begin{array}{c}\text { Altura } \\
\text { (cm) }\end{array}$ & $\begin{array}{c}\mathrm{AC} \\
\left(\mathrm{m}^{2}\right)\end{array}$ & $\begin{array}{c}\text { DAS } \\
(\mathrm{mm})\end{array}$ & $\begin{array}{c}\text { Altura } \\
\text { (cm) }\end{array}$ \\
\hline Sabiá & $98,6 \mathrm{a}$ & $40,8 \mathrm{a}$ & $232,1 \mathrm{a}$ & $5,9 \mathrm{a}$ & $69,5 \mathrm{a}$ & $436,6 \mathrm{a}$ \\
\hline E. camaldulensis + sabiá & $97,2 \mathrm{a}$ & $40,8 \mathrm{a}$ & $214,7 \mathrm{a}$ & $5,1 \mathrm{ab}$ & $66,0 \mathrm{ab}$ & $388,7 \mathrm{ab}$ \\
\hline E. pellita + sabiá & $100,0 \mathrm{a}$ & $38,4 \mathrm{a}$ & $208,5 \mathrm{a}$ & $3,8 \mathrm{~b}$ & $57,7 \mathrm{~b}$ & $366,3 \mathrm{~b}$ \\
\hline E. tereticornis + sabiá & 95,8 a & $37,7 \mathrm{a}$ & 204,0 a & $4,5 \mathrm{ab}$ & $61,1 \mathrm{ab}$ & $388,5 \mathrm{ab}$ \\
\hline E. robusta + sabiá & $97,2 \mathrm{a}$ & $35,5 \mathrm{a}$ & $202,1 \mathrm{a}$ & $4,5 \mathrm{ab}$ & $62,1 \mathrm{ab}$ & $385,4 \mathrm{ab}$ \\
\hline Média Geral & 97,8 & 38,6 & 212,3 & 4,8 & 63,3 & 393,1 \\
\hline $\mathrm{CV} \%$ & 3,6 & 8,3 & 6,6 & 17,1 & 6,72 & 8,97 \\
\hline
\end{tabular}

Médias na coluna $(\mathrm{n}=4)$ seguidas por letras iguais não diferem estatisticamente pelo teste de Tukey $(\alpha=0,05)$. CV\%= coeficiente de variação, $\mathrm{DAS}=$ diâmetro a altura do solo, $\mathrm{AC}=$ área de copa.

Segundo Paulino et al. (2002), o plantio de sabiá apresentou alta sobrevivência e crescimento em pastagem degradada, apresentando, aos 24 meses, $87 \%$ de sobrevivência e 3,26 m de altura, resultados inferiores aos constatados neste estudo.

O sistema de plantio não afetou a produção de raízes finas do sabiá aos 24 meses de idade (Tabela 4). Considerando os resultados das características dendrométricas, era de se esperar que, quando consorciado com E. pellita, o sabiá apresentasse maior área e comprimento de raízes finas, como reflexo da competição interespecífica, uma vez que a menor disponibilidade de recursos, normalmente, estimula a produção dessas raízes (KEITH et al., 1997; GIARDINA; RYAN, 2002; MISRA et al., 1998; SCHMID; KAZDA, 2002; GONÇALVEZ, 1995), fato observado para E. robusta consorciado com Mimosa caesalpiniifolia (MENDONÇA, 2006). Entretanto, embora o sabiá tenha apresentado maior área e comprimento de raízes finas quando consorciado com $E$. pellita, a análise estatística comprovou que essa diferença não é significativa.

Tabela 4. Área e comprimento de raízes finas do sabiá aos 24 meses após o plantio.

Table 4. Area and length of fine roots of sabia at the 24 months after the plantation.

\begin{tabular}{lcc} 
Tratamento & $\begin{array}{c}\text { Área } \\
\left(\mathbf{c m}^{\mathbf{2}} \mathbf{d m}^{\mathbf{3}} \mathbf{)}\right.\end{array}$ & $\begin{array}{c}\text { Comprimento } \\
\left(\mathbf{c m . d m} \mathbf{3}^{\mathbf{3}}\right.\end{array}$ \\
\hline Sabiá & $33,1 \mathrm{a}$ & $1289,9 \mathrm{a}$ \\
E. camaldulensis + sabiá & $33,1 \mathrm{a}$ & $1063,4 \mathrm{a}$ \\
E. pellita + sabiá & $50,2 \mathrm{a}$ & $1510,8 \mathrm{a}$ \\
E. tereticornis + sabiá & $28,6 \mathrm{a}$ & $851,5 \mathrm{a}$ \\
E. robusta + sabiá & $32,9 \mathrm{a}$ & $1049,6 \mathrm{a}$ \\
Média Geral & 35,6 & 1153,0 \\
\hline CV\% & 30,6 & 33,8 \\
\hline
\end{tabular}

Médias na coluna $(n=4)$ seguidas por letras iguais não diferem estatisticamente pelo teste de Tukey $(\alpha=0,05)$. CV\%= coeficiente de variação.

\section{Biomassa e quantidade de nutrientes fornecidos pela poda das plantas de sabiá}

As equações para estimação da biomassa seca de folhas, galhos e do total, do material proveniente da poda do sabiá, para 12 e 24 meses, estão apresentadas na tabela 5.

As equações ajustadas para estimação da biomassa resultante da poda do sabiá têm aplicação prática no manejo da fertilidade do solo, considerando que o sabiá desempenha a função de adubo verde. 
Conhecendo a biomassa seca e a concentração de nutrientes nos tecidos do material podado, estima-se a quantidade de nutrientes fornecida pela poda.

Tabela 5. Equações para estimação de biomassa seca de folha, galho e total, aos 12 e 24 meses.

Table 5. Equations for estimating dry biomass of leaf, branch and total, at the 12 and 24 months.

\begin{tabular}{|c|c|c|c|}
\hline \multirow{2}{*}{ Parte da planta } & \multicolumn{3}{|c|}{12 meses } \\
\hline & Equação & $\mathbf{R}^{2 *}$ & CV\% \\
\hline Folha & $\mathrm{MS}=-0,021+0,091 \mathrm{AC}+0,0000209 \mathrm{DAS} * \mathrm{H}+\mathrm{e}_{\mathrm{i}}$ & 78,2 & 21,45 \\
\hline Galho & $\mathrm{MS}=0,0949 \mathrm{AC}^{1,2012}+\mathrm{e}_{\mathrm{i}}$ & - & 57,4 \\
\hline Total & $\mathrm{MS}=-0,46+0,012 \mathrm{DAS}+0,26 \mathrm{AC}+\mathrm{e}_{\mathrm{i}}$ & 83,8 & 22,1 \\
\hline \multirow{2}{*}{ Parte da planta } & \multicolumn{3}{|l|}{ ( } \\
\hline & Equação & $\overline{\mathbf{R}^{2}}$ & CV\% \\
\hline Folha & $\mathrm{MS}=0,4508+0,00005 \mathrm{DAS} * \mathrm{H}+\mathrm{e}_{\mathrm{i}}$ & 42,7 & 24,3 \\
\hline Galho & $\mathrm{MS}=-1,23+0,037 \mathrm{DAS}+0,0037 \mathrm{H}+\mathrm{e}_{\mathrm{i}}$ & 63,4 & 24,1 \\
\hline Total & $\mathrm{MS}=0,0355 \mathrm{DAS}^{1,1558}+\mathrm{e}_{\mathrm{i}}$ & - & 20,5 \\
\hline
\end{tabular}

Aos 12 e 24 meses, a biomassa média por planta de folhas, galhos e total proveniente da poda do sabiá foi maior no plantio puro de sabiá e menor no plantio consorciado de E. pellita + sabiá (Tabela 6). Assim, quando consorciado com E. pellita, o sabiá produz menos biomassa na parte aérea em relação ao plantio puro.

Tabela 6. Biomassa média $(\mathrm{kg})$ por planta do material proveniente da poda de sabiá.

Table 6. Average biomass $(\mathrm{kg})$ for plant of the material proceeding from the pruning of sabia.

\begin{tabular}{lcccccc}
\hline \multirow{2}{*}{ Tratamento } & \multicolumn{3}{c}{ 12 meses } & \multicolumn{3}{c}{ 24 meses } \\
\cline { 2 - 7 } & Folha & Galho & Total & Folha & Galho & Total \\
\hline Sabiá & $0,72 \mathrm{a}$ & $0,83 \mathrm{a}$ & $1,56 \mathrm{a}$ & $1,86 \mathrm{a}$ & $2,92 \mathrm{a}$ & $4,80 \mathrm{a}$ \\
E. camaldulensis + sabiá & $0,64 \mathrm{ab}$ & $0,69 \mathrm{ab}$ & $1,37 \mathrm{ab}$ & $1,64 \mathrm{ab}$ & $2,62 \mathrm{ab}$ & $4,53 \mathrm{ab}$ \\
E. pellita+sabiá & $0,50 \mathrm{~b}$ & $0,48 \mathrm{~b}$ & $0,99 \mathrm{~b}$ & $1,44 \mathrm{~b}$ & $2,23 \mathrm{~b}$ & $3,89 \mathrm{~b}$ \\
E. tereticornis + sabiá & $0,55 \mathrm{ab}$ & $0,59 \mathrm{ab}$ & $1,16 \mathrm{ab}$ & $1,55 \mathrm{~b}$ & $2,43 \mathrm{ab}$ & $4,14 \mathrm{ab}$ \\
E. robusta + sabiá & $0,55 \mathrm{ab}$ & $0,60 \mathrm{ab}$ & $1,15 \mathrm{ab}$ & $1,56 \mathrm{ab}$ & $2,46 \mathrm{ab}$ & $4,22 \mathrm{ab}$ \\
\hline CV\% & 13,89 & 21,63 & 18,67 & 9,10 & 10,14 & 7,63 \\
\hline
\end{tabular}

Médias na coluna $(n=4)$ seguidas por letras iguais não diferem estatisticamente pelo teste de Tukey $(\alpha=0,05)$. CV\%= coeficiente de variação

A produção de biomassa seca por hectare das folhas, galhos e total, aos 12 e 24 meses, obtida mediante utilização das equações ajustadas, conforme a expectativa, foi maior para o plantio puro, certamente devido ao maior número de plantas de sabiá nesse tratamento. Ressalte-se que, entre os tratamentos consorciados, não foram detectadas diferenças para essas variáveis. Comportamento semelhante foi observado para a quantidade de nutrientes, de $\mathrm{C}$ e de $\mathrm{Na}$ fornecidos pelo material da poda aos 12 meses, exceto para o $\mathrm{S}$ nas folhas e $\mathrm{Mn}$ nos galhos, que não diferiu entre o plantio puro e o consorciado.

Foram obtidos intervalos de confiança $(\alpha=0,05)$ para as quantidades de biomassa seca de folhas e de galhos, de nutrientes, de $\mathrm{C}$ e de $\mathrm{Na}$ fornecidas pela poda de plantas de sabiá nos plantios puro e consorciados, sem discriminar as espécies de eucalipto (Tabelas 7, 8 e 9).

O potencial do sabiá como espécie adubadeira é comprovado nos estudos desenvolvidos por Andrade et al. (2000) e Costa et al. (2004). A serapilheira produzida pelas plantas de sabiá é rica em nutrientes e apresenta elevada velocidade de decomposição (ANDRADE et al., 2000). Outro aspecto positivo dessa espécie é a elevada produção de biomassa, o que está fortemente associado ao aporte de nutrientes pela serapilheira (COSTA et al., 2004). Entretanto, até o momento, não foram encontradas na literatura informações sobre a quantidade de biomassa seca e de nutrientes fornecida por podas freqüentes de sabiá. 
Tabela 7. Conteúdo de $\mathrm{C}$ e biomassa seca proveniente da poda de plantas de sabiá para folha, galho e total aos 12 e 24 meses, para plantios puros e consorciados com eucalipto.

Table 7. Content of $\mathrm{C}$ and dry biomass coming from pruning of plants of sabia for leaf, branch and total at the 12 and 24 months, for pure and joined plantation with eucalypt.

\begin{tabular}{|c|c|c|c|c|c|c|c|c|c|c|c|c|c|c|c|c|}
\hline \multirow{4}{*}{ Parâmetros } & \multicolumn{10}{|c|}{12 meses } & \multicolumn{6}{|c|}{24 meses } \\
\hline & \multicolumn{6}{|c|}{ Massa seca tha ${ }^{-1}$} & \multicolumn{4}{|c|}{ C tha ${ }^{-1}$} & \multicolumn{6}{|c|}{ Massa seca t ha ${ }^{-1}$} \\
\hline & \multicolumn{3}{|c|}{ Sabiá } & \multicolumn{3}{|c|}{ Sabiá+eucalipto } & \multicolumn{2}{|c|}{ Sabiá } & \multicolumn{2}{|c|}{ Sabiá+eucalipto } & \multicolumn{3}{|c|}{ Sabiá } & \multicolumn{3}{|c|}{ Sabiá+eucalipto } \\
\hline & $\mathbf{F}$ & G & $\mathbf{T}$ & $\mathbf{F}$ & G & $\mathbf{T}$ & $\mathbf{F}$ & $\mathbf{G}$ & $\mathbf{F}$ & G & $\mathbf{F}$ & G & $\mathbf{T}$ & $\mathbf{F}$ & $\mathbf{G}$ & $\mathbf{T}$ \\
\hline Média & 1,7 & 1,9 & 3,6 & 0,63 & 0,67 & 1,3 & 0,82 & 0,8 & 0,30 & 0,29 & 4,4 & 6,9 & 11,3 & 1,81 & 2,9 & 4,9 \\
\hline $\mathrm{S}$ & 0,4 & 0,6 & 0,9 & 0,17 & 0,21 & 0,4 & 0,17 & 0,2 & 0,08 & 0,10 & 0,2 & 0,4 & 0,8 & 0,20 & 0,4 & 0,6 \\
\hline EPM & 0,2 & 0,3 & 0,5 & 0,04 & 0,05 & 0,1 & 0,08 & 0,1 & 0,02 & 0,02 & 0,1 & 0,2 & 0,4 & 0,05 & 0,1 & 0,1 \\
\hline LI & 1,1 & 1,0 & 2,1 & 0,54 & 0,55 & 1,1 & 0,55 & 0,5 & 0,26 & 0,24 & 4,0 & 6,3 & 10,1 & 1,70 & 2,7 & 4,6 \\
\hline LS & 2,3 & 2,8 & 5,1 & 0,72 & 0,78 & 1,5 & 1,08 & 1,2 & 0,35 & 0,34 & 4,8 & 7,5 & 12,6 & 1,92 & 3,0 & 5,2 \\
\hline
\end{tabular}

$\mathrm{S}=$ desvio padrão, $\mathrm{EPM}=$ erro padrão da média, $\mathrm{CV} \%=$ coeficiente de variação, $\mathrm{LI}=$ limite inferior do intervalo de confiança, $\mathrm{LS}=$ limite superior do intervalo de confiança $(\alpha=0,05), \mathrm{F}=$ biomassa seca de folha, $\mathrm{G}=$ biomassa seca de galho, $\mathrm{T}=$ biomassa seca total.

Tabela 8. Macronutrientes e $\mathrm{Na}\left(\mathrm{kg} \mathrm{ha}^{-1}\right)$ fornecidos pela biomassa seca da poda de plantas de sabiá em plantios puros e consorciados com eucalipto, aos 12 meses.

Table 8. Macronutrients and $\mathrm{Na}\left(\mathrm{kg} \mathrm{ha}^{-1}\right)$ supplied by dry biomass of pruning of plants of sabia in pure and joined plantation with eucalypt, at the 12 months.

\begin{tabular}{|c|c|c|c|c|c|c|c|c|c|c|c|c|c|c|}
\hline \multirow{3}{*}{ Parâmetros } & \multicolumn{14}{|c|}{ Folha } \\
\hline & \multicolumn{7}{|c|}{ Sabiá } & \multicolumn{7}{|c|}{ Sabiá + Eucalipto } \\
\hline & $\mathbf{N}$ & $\mathbf{P}$ & $\mathbf{K}$ & $\mathbf{C a}$ & Mg & $\mathbf{S}$ & $\mathbf{N a}$ & $\mathbf{N}$ & $\mathbf{P}$ & $\mathbf{K}$ & $\mathrm{Ca}$ & Mg & $\mathbf{S}$ & $\mathbf{N a}$ \\
\hline Média & 37,1 & 2,7 & 11,3 & 26,4 & 4,9 & 46,9 & 0,4 & 13,3 & 1,0 & 3,9 & 8,3 & 1,8 & 50,5 & 0,1 \\
\hline $\mathrm{S}$ & 8,8 & 0,4 & 2,7 & 11,7 & 1,1 & 13,7 & 0,1 & 3,6 & 0,3 & 1,2 & 2,9 & 0,8 & 18,0 & 0,1 \\
\hline EPM & 4,4 & 0,2 & 1,3 & 5,9 & 0,5 & 6,8 & 0,04 & 0,9 & 0,1 & 0,3 & 0,7 & 0,2 & 4,5 & 0,0 \\
\hline $\mathrm{CV} \%$ & 23,7 & 16,7 & 23,6 & 44,4 & 22,3 & 29,2 & 22,2 & 26,8 & 29,8 & 29,9 & 35,4 & 42,7 & 35,6 & 41,6 \\
\hline LI & 23,1 & 2,0 & 7,1 & 7,7 & 3,1 & 25,1 & 0,2 & 11,4 & 0,8 & 3,3 & 6,7 & 1,4 & 40,9 & 0,1 \\
\hline LS & 51,1 & 3,4 & 15,6 & 45,0 & 6,6 & 68,6 & 0,5 & 15,2 & 1,1 & 4,5 & 9,8 & 2,2 & 60,1 & 0,2 \\
\hline \multirow{3}{*}{ Parâmetros } & \multicolumn{14}{|c|}{ Galho } \\
\hline & \multicolumn{7}{|c|}{ Sabiá } & \multicolumn{7}{|c|}{ Sabiá + Eucalipto } \\
\hline & $\mathbf{N}$ & $\mathbf{P}$ & $\mathbf{K}$ & $\mathrm{Ca}$ & Mg & $\mathbf{S}$ & $\mathbf{N a}$ & $\mathbf{N}$ & $\mathbf{P}$ & $\mathbf{K}$ & $\mathrm{Ca}$ & Mg & $\mathbf{S}$ & $\mathbf{N a}$ \\
\hline Média & 9,9 & 1,9 & 17,3 & 12,2 & 1,9 & 4,1 & 1,01 & 3,3 & 0,7 & 2,8 & 12,2 & 0,5 & 0,8 & 0,2 \\
\hline $\mathrm{S}$ & 2,9 & 0,7 & 11,9 & 4,3 & 0,9 & 3,0 & 0,62 & 0,9 & 0,3 & 0,9 & 4,3 & 0,1 & 0,3 & 0,1 \\
\hline EPM & 1,5 & 0,3 & 6,0 & 2,1 & 0,5 & 1,5 & 0,31 & 0,2 & 0,1 & 0,2 & 2,1 & 0,04 & 0,1 & 0,03 \\
\hline $\mathrm{CV} \%$ & 29,4 & 33,6 & 68,7 & 35,1 & 50,7 & 72,0 & 61,41 & 28,6 & 45,3 & 33,5 & 35,1 & 27,4 & 34,7 & 49,0 \\
\hline LI & 5,3 & 0,9 & $-1,6$ & 5,4 & 0,4 & $-0,6$ & 0,02 & 2,8 & 0,5 & 2,3 & 5,4 & 0,5 & 0,7 & 0,2 \\
\hline LS & 14,5 & 3,0 & 36,3 & 19,0 & 3,3 & 8,8 & 2,00 & 3,8 & 0,9 & 3,2 & 19,0 & 0,6 & 1,0 & 0,3 \\
\hline
\end{tabular}

$\mathrm{S}=$ desvio padrão, EPM= Erro padrão da média, $\mathrm{CV} \%=$ coeficiente de variação, $\mathrm{LI}=$ limite inferior do intervalo de confiança, $\mathrm{LS}=$ limite superior do intervalo de confiança $(\alpha=0,05)$.

É importante ressaltar que a quantidade de biomassa seca e de nutrientes fornecida por resíduos vegetais é influenciada pelas condições locais, idade da planta e época do ano. Andrade et al. (2000) encontraram, na serapilheira de sabiá, menores quantidades de $\mathrm{N}$ e $\mathrm{P}$ em relação às encontradas por Costa et al. (2004). Santiago (2005) encontrou concentrações superiores de N, P e K na biomassa seca proveniente da poda de sesbânia aos 22 meses de idade, em relação aos 10 meses (COUTINHO, 2003).

\section{CONCLUSÕES}

Considerando a reabilitação do solo das cavas de extração de argila, entre os tratamentos testados o mais promissor é o consórcio entre E. robusta + sabiá, pois, além de proporcionar aumento no teor de C no solo, contribui para redução da salinidade. 
Em todos os sistemas de plantio testados, houve redução de $\mathrm{K}$ no solo da cava de extração de argila, o que indica que para o estabelecimento de plantios nessas áreas, com as espécies avaliadas, é necessário o emprego de um sistema de adubação para o fornecimento desse nutriente.

Mimosa caesalpiniifolia (sabiá) apresenta menor crescimento quando consorciado com $E$. pellita, indicando que sofre o efeito da competição interespecífica imposta por E. pellita.

Tabela 9. Micronutrientes $\left(\mathrm{g} \mathrm{ha}^{-1}\right)$ fornecidos pela biomassa seca da poda de plantas de sabiá em plantios puros e consorciados com eucalipto, aos 12 meses.

Table 9. Macronutrients $\left(\mathrm{g} \mathrm{ha}^{-1}\right)$ supplied by dry biomass of pruning of plants of sabia in pure and joined plantation with eucalypt, at the 12 months.

\begin{tabular}{|c|c|c|c|c|c|c|c|c|}
\hline \multirow{3}{*}{ Parâmetros } & \multicolumn{8}{|c|}{ Folha } \\
\hline & \multicolumn{4}{|c|}{ Sabiá } & \multicolumn{4}{|c|}{ Sabiá + Eucalipto } \\
\hline & $\mathbf{C u}$ & Zn & Mn & $\mathbf{F e}$ & $\mathbf{C u}$ & $\mathbf{Z n}$ & Mn & $\mathbf{F e}$ \\
\hline $\mathrm{S}$ & 3,9 & 5,2 & 22,5 & 97,8 & 1,5 & 3,2 & 18,3 & 75,6 \\
\hline EPM & 2,0 & 2,6 & 11,2 & 48,9 & 0,4 & 0,8 & 4,6 & 18,9 \\
\hline $\mathrm{cv} \%$ & 37,9 & 20,2 & 36,0 & 28,5 & 39,5 & 32,9 & 68,3 & 52,4 \\
\hline LS & 16,6 & 33,8 & 98,2 & 498,5 & 4,7 & 11,4 & 36,6 & 184,7 \\
\hline \multirow{3}{*}{ Parâmetros } & \multicolumn{8}{|c|}{ Galho } \\
\hline & \multicolumn{4}{|c|}{ Sabiá } & \multicolumn{4}{|c|}{ Sabiá + Eucalipto } \\
\hline & $\mathrm{Cu}$ & Zn & Mn & Fe & $\mathbf{C u}$ & $\mathbf{Z n}$ & Mn & $\mathbf{F e}$ \\
\hline Média & 10,2 & 24,8 & 23,9 & 161,3 & 3,5 & 3,5 & 5,9 & 51,9 \\
\hline LI & 4,6 & $-7,4$ & $-9,9$ & 38,9 & 2,8 & 2,7 & 3,2 & 40,3 \\
\hline LS & 15,8 & 57,0 & 57,8 & 283,7 & 4,1 & 4,3 & 8,6 & 63,5 \\
\hline
\end{tabular}

\section{REFERÊNCIAS}

ALVARENGA, M. I. N.; DAVIDE, A. C. Características físicas e químicas de um latossolo vermelho escuro e a sustentabilidade de agroecossistemas. Revista Brasileira de Ciência do Solo, Campinas, v. 23, n. 4, p. 933-942, 1999.

ANDRADE, A. G., COSTA, G. S., FARIA, S. M. Deposição e decomposição da serrapilheira em povoamentos de Mimosa caesalpiniifolia, Acacia mangium e Acacia holosericea com quatro anos de idade em plantossolo. Revista Brasileira de Ciência do Solo, Campinas, v. 24, n. 4 p. 777-785, 2000.

CAMPOS, H. Estatística experimental não paramétrica. 3. ed. Piracicaba: ESALQ, 1979. 373 p.

COSTA, G. S.; FRANCO, A. A.; DAMASCENO, R. N.; FARIA, S. M. Aporte de nutrientes pela serrapilheira em uma área degradada e revegetada com leguminosas arbóreas. Revista Brasileira de Ciência do Solo, Campinas, v. 28, n. 5, p. 919-927, 2004.

COUTINHO, M. P. Crescimento de Sesbania virgata (Cav.) Pers. em cavas de extração de argila. 54 p. Dissertação (Mestrado em Produção Vegetal) - Universidade Federal do Norte Fluminense, Campos dos Goytacazes, 2003

EMBRAPA. Manual de métodos de análises de solo. 2. ed, Rio de Janeiro: Centro Nacional de Pesquisa de Solos, 1997. 212 p. (Documentos; n. 1).

FORRESTER, D. I.; BAUHUS, J.; COWIE, A. L. On the success and failure of mixed species tree plantations: lessons learned from a model system of Eucalyptus globulus and Acacia mearnsii. Forest Ecology and Management, Amsterdam, v. 209, n. 1-2, p. 147-155, 2005. 
FORRESTER, D. I.; BAUHUS, J.; KHANNA, P. K. Growth dynamics in a mixed-species plantation of Eucalyptus globulus and Acacia mearnsii. Forest Ecology and Management, Amsterdam, v. 193, n. 1-2, p. 81-95, 2004.

GAMA RODRIGUES, A. C.; BARROS, N. F.; MENDONÇA, E. S. Alterações edáficas sob plantios puros e misto de espécies florestais nativas do sudeste da Bahia, Brasil. Revista Brasileira de Ciência do Solo, Campinas, v. 23, n. 3, p. 581-592, 1999.

GARAY, I.; KINDEL, A.; CARNEIRO, R.; FRANCO, A. A.; BARROS, E.; ABBADIE, L. Comparação da matéria orgânica e de outros atributos do solo entre as plantações de Acacia mangium e Eucalyptus grandis. Revista Brasileira de Ciência do Solo, Campinas, v. 27, n. 4, p. 705-712, 2003.

GIARDINA, C. P.; RYAN, M. G. Total belowground carbon allocation in a fast-growing Eucalyptus plantation estimate using a carbon balance approach. Ecosystems, New York, v. 5, n. 5, p. 487-499, 2002.

GONÇALVES, C. de A., FERNANDES, M. M., ANDRADE, A. M. de Celulose e Carvão vegetal de Mimosa Caesalpiniaefolia Benthan (Sabiá). Floresta e Ambiente, Seropedica, v. 6, n. 1, p. 51-58, 1999.

GONÇALVEZ, J. L. M. Características do sistema radicular de absorção do Eucalyptus grandis sob diferentes condições edáficas: I Distribuição de raízes nas camadas de solo. In: CONGRESSO BRASILEIRO DE CIÊNCIAS DO SOLO, 25., 1995, Viçosa. Anais do... Viçosa, MG: Sociedade Brasileira de Ciência do Solo, 1995. p. 876-878.

JAIYEOBA, I. A. Amelioration of soil fertility by woody perennials in cropping fields: evaluation of three species in the semi-arid zone of Nigeria. Journal of Arid Environments, London, v. 33, n. 4, p. 473-482, 1996.

JONES JÚNIOR., J. B., WOLF, B., MILLS, H. A. Plant analysis handbook: a practical sampling, preparation, analysis and interpretation guide. Athens (USA): Micro - Macro, 1991. 213p.

JÚNIOR LEAL, G.; SILVA, J. A. da Proposta de manejo florestal sustentado do sabiá (Mimosa caesalpiniifolia). Crato: IBAMA, 1999. 15 p., 1999. (Boletim Técnico; n. 3).

KEITH, H.; RAISON, R. J.; JACOBSEN, K. L. Allocation of carbon in mature eucalipt forest and some effects of soil phosphorus availability. Plant and Soil, The Hague, v. 196, n. 1, p. 81-99, 1997.

KUMAR, B. M.; KUMAR, S. S.; FISHER, R. F. Intercropping Teak with Leucena increases tree growth and modifies soil characteristics. Agroforestry Systems, Dordrecht, v. 42, n. 1, p. 81-89, 1998.

MAFRA, A. L., MIKLÓS, A. A. W. de, VOCURCA, H. L., HARKALY, A. H., MENDONZA, E. (1998) Adição de nutrientes ao solo em sistema agroflorestal do tipo "cultivo em aléias" e em cerrado na região de Botucatu, SP. Scientia Forestalis, Piracicaba, n. 54, p. 41-54, 1998.

Malavolta, E., VITTI, G. C., Oliveira, S. A. Avaliação do estado nutricional das plantas: princípio e aplicações. 2. ed. Piracicaba: Potafo, 1997. 319p.

MELO, V. F.; CORRÊA, G. F.; RIBEIRO, A. N.; MASCHIO, P. A. Cinética de liberação de potássio e magnésio pelos minerais da fração argila de solos do Triângulo Mineiro. Revista Brasileira de Ciência do Solo, Campinas, v. 29, n. 4, p. 533-545, 2005.

MELO, V. F.; BARROS, N. F.; COSTA, L. M.; NOVAIS, R. F.; FONTES, M. P. F. Formas de potássio e de magnésio em solos do Rio Grande do Sul, e sua relação com o conteúdo na planta e com a produção em plantios de eucalipto. Revista Brasileira de Ciência do Solo, Campinas, v. 19, n. 2, p. 165-171, 1995.

MENDONÇA, A. V. R. Reabilitação de cavas de extração de argila e tolerância de espécies florestais a salinidade. Tese (Tese Doutorado em Produção Vegetal) - Universidade Federal do Norte Fluminense, Campos dos Goytacazes, 2006,

MISHRA, A.; SHARMA, S. D.; KHAN, G. H. Improvement in physical and chemical properties of sodic soil by 3, 6 and 9 yers old plantations of Eucalyptus tereticornis: Biorejuvenation of sodic soil. Forest Ecology and Management, Amsterdam, v. 184, n. 1-3, p. 115-124, 2003. 
MISRA, R. K.; TURNBUll, C. R. A.; CROMER, R. N.; GIBBONS, A. K.; LA SAlA, A. V.; BALLARD, L. M. Below and above ground growth of Eucalyptus nitens in a young plantation. II Nitrogen and Phosphorus. Forest Ecology and Management, Amsterdam, v. 106, n. 2-3, p. 295-306, 1998.

NOBLE, A. D.; ZENNECK, I.; RANDALL, P. J. Leaf litter ash alkalinity and neutralization of soil acidity. Plant Soil, The Hague, v. 179, n. 2, p. 293-302, 1996.

PAUlinO, G. M.; SANTOS, M. L. dos; FROUDE, L. C. M.; GAMA RODRIGUES, A. C.; FRNACO, A. A.; GAMA RODRIGUES, E. F. Estudo da contribuição de espécies florestais para a recuperação de terras degradadas por pastagem em Conceição de Macabu, RJ. In: SIMPÓSIO NACIONAL DE RECUPERAÇÃO DE ÁREAS DEGRADADAS, 5., 2002, Belo horizonte. Anais do... Belo Horizonte: SOBRADE, 2002. p. 508-509.

PARROTA, J. A. Productivity, nutrient cycling, and succession in single- and mixed-species plantations of Casuarina equisetifolia, Eucalyptus robusta, and Leucaena leucocephala in Puerto Rico. Forest Ecology and Management, Amsterdam, v. 124, n. 1, p. 45-77, 1999.

RAMOS, I. de S., ALEXANDRE, J., ALVES, M. da G., BARROSO, J. A., TEIXEIRA, L. S., CORREA, F. de P. Dimensionamento da indústria cerâmica em Campos dos Goytacazes, RJ. In: CONGRESSO BRASILEIRO DE CERÂMICA, 47, João Pessoa-PB, 2003. Anais do... São Paulo : TECART, 2003. 1 CD-ROM.

RAMOS, D. P., MANZATTO, C. V. Estudos de solos para ordenamento do uso cerâmico das argilas da Baixada Campista, estado do Rio de Janeiro. Rio de Janeiro: Embrapa Solos, 2003. 19 p. (Documentos, n. 52).

SANTIAGO, A. R. Eucalipto em plantios puros e consorciados com Sesbânia na reabilitação de cavas de extração de argila. 77 p. Dissertação (Mestrado em Produção Vegetal) - Universidade Estadual do Norte Fluminense, Campos dos Goytacazes, 2005

SCHIAVO, J. A. Revegetação de áreas degradadas pela extração de argila, com espécies micorrizadas de Acacia mangium, Sesbania virgata e Eucalyptus camaldulensis. 117 p. Tese (Doutorado em Produção Vegetal) - Universidade Estadual do Norte Fluminense (UENF) Campos dos Goytacazes, 2005.

SCHMID, I.; KAZDA, M. Root distribution of Norway spruce in monospecific and mixed stands on different soils. Forest Ecology and Management, Amsterdam, v. 159, n. 1-2, p. 37-47, 2002.

SNEDECOR, W., COCHRAN, W. G. Statistical methods. 8. ed. Ames: Iowa State University Press, 1989. $502 \mathrm{p}$.

SONG, S. K.; HUANG, P. M. Dynamics of potassium release from potassium-bearing minerals as influenced by oxalic and citric acids. Soil Science Society of America Journal, Madison, v. 52, p. 383390, 1988.

TOKY, O. P.; SINGH, V. Nutrient dynamics in Leucaena, Acacia and Eucalyptus, short rotation, high density (energy) plantations in arid India. Journal of Arid Environments, London, v. 31, n. 4, p. 401413, 1995.

TOKY, O. P.; RAMAKRISHNAN, P. S. Role off bamboo (Dendrocalamus hamiltonii Ness and Arn.) in conservation of potassium during slash and burn agriculture (jhum) in north-eastern India, Journal of Tree Sciences, New Delhi, v. 1, p. 17-26, 1982.

VALICHESKI, R. R. Avaliação técnica e econômica da reutilizaçao de áreas de extração de argila em Campos dos Goytacazes-RJ. 132 p. Dissertação (Mestrado em Produção Vegetal) - Universidade Estadual do Norte Fluminense, Campos dos Goytacazes, 2004

VEZZANI, F. M.; TEDESCO, M. J.; BARROS, N. F. Alterações dos nutrientes no solo e plantas em consórcio de Eucalipto e Acácia negra. Revista Brasileira de Ciência do Solo, Campinas, v. 25, n.1 , p. $225-231,2001$.

ZINN, Y. L.; RESCK, D. V. S.; SILVA, J. E. da. Soil organic carbon as affected by afforestation with Eucalyptus and Pinus in Cerrado region of Brazil. Forest Ecology and Management, Amsterdam,v. 166, n. 1-3, p. 285-294, 2002. 